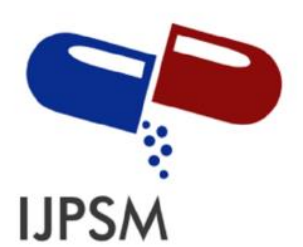

Kamini Kashyap, International Journal of Pharmaceutical Sciences and Medicine (IJPSM), Vol.6 Issue. 7, July- 2021, pg. 102-127

ISSN: 2519-9889

Impact Factor: $\mathbf{3 . 4 2 6}$

\title{
ARCHAEOSOMES: REVOLUTIONARY TECHNIQUE FOR BOTH CELL-BASED AND DRUG-BASED DELIVERY APPLICATIONS
}

\author{
Kamini Kashyap* \\ Department of Pharmaceutics, School of Pharmacy, Chouksey College of Engineering, NH-49, Masturi - \\ Jairamnagar Road, Lalkhadan, Bilaspur 495004, Chhattisgarh, India
}

DOI: 10.47760/ijpsm.2021.v06i07.008

\section{*Corresponding Author:}

E-Mail: kashyapkamini127@gmail.com

Phone: +91-9109932032

\begin{abstract}
Archaeosomes, or liposomes formed with one or more ether lipids specific to the Archaeobacteria domain, are a new kind of liposome discovered in Archaea. Archaeol (diether) and/or caldarchaeol (tetraether) are the basic structures of Achaean-type lipids. Archaeosomes may be made using conventional methods (hydrated film sonicated, extrusion, and detergent dialysis) at any temperature in the physiological range or below, allowing thermally stable chemicals to be encapsulated. Its stability is influenced by a variety of physiological and environmental variables. For cancer vaccines, Chagas disease, proteins and peptides, gene delivery, antigen delivery, and delivery of natural antioxidant chemicals, archaeosomes are extensively utilized as drug delivery systems. The main goal of this review paper was to look at the uses of this novel carrier system in the pharmaceutical sector.
\end{abstract}

Keywords: Archaeobacteria, Archaeosomes, Cell Delivery, Drug Delivery, Gene Delivery, Formulations

\section{INTRODUCTION}

Archaeosomes are liposomes produced with one or more ether lipids that are unique to the Archaeobacteria domain, as well as vesicles generated from a variety of lipids that include archaea bacterial ether lipids. The lipid membrane of archaeosomes can be a bilayer (if made 


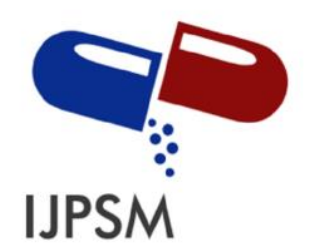

Kamini Kashyap, International Journal of Pharmaceutical Sciences and Medicine (IJPSM), Vol.6 Issue. 7, July- 2021, pg. 102-127

ISSN: 2519-9889

Impact Factor: 3.426

exclusively from monopole archaeol lipids or lipid mixtures containing archaeols and nonarchaeobacterial monopolar lipids), a monolayer (if made exclusively from bipolar caldarchaeol lipids), or a combination of mono and bilayers (if made exclusively from bipolar caldarchaeol lipids) (if made from caldarchaeol lipids and archaeols or other monopolar lipids). Archaeosomes are a new kind of liposome that is made using one or more ether lipids present in Archaea. Archaeol (diether) and/or caldarchaeol (tetraether) core structures are found in lipids of the Achaean type. The architecture of archaeosomes promotes the existence of both hydrophobic and hydrophilic domains, allowing them to entrap both hydrophilic and hydrophobic molecules, which is particularly useful for encapsulation and drug administration. The utilization of synthetically produced lipids with the distinctive structural features of archaeobacterial ether lipids, such as branching phytanyl chains linked through ether bonds at sn-2, 3 glycerol carbons, is included in the concept of archaeosomes. The lipid membrane of archaeosomes can be found as a bilayer if made entirely of monopolar archaeol (diether) lipids, or as a monolayer if made entirely of bipolar caldarchaeol (tetraether) lipids, or as a mixture of monolayers and bilayers if made entirely of caldarchaeol lipids in addition to archaeol lipids or standard bilayer-forming The wide variety of lipid structures reflects Archaea's requirement to alter their basic lipid structures to maintain membrane function even in disruptive environments [1].

A typical characteristic should be particularly useful for the preparation of highly stable archaeosomes [2]:

- The branching methyl groups assist to decrease both crystallization (membrane lipids in the liquid crystalline state at ambient temperature, membrane permeability, membrane lipids in the liquid crystalline state at ambient temperature, and steric hindrance of the methyl side groups). 


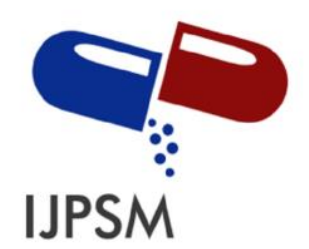

Kamini Kashyap, International Journal of Pharmaceutical Sciences and Medicine (IJPSM), Vol.6 Issue. 7, July- 2021, pg. 102-127

ISSN: 2519-9889 Impact Factor: 3.426

- The saturated alkyl chains would provide stability against oxidative destruction, and the glycerol backbone's unique stereochemistry (opposite to mesophilic species) would guarantee resistance to phospholipases produced by other organisms.

- The bipolar lipids span the membranes and improve the membrane's stability.

- Adding cyclic structures (especially five-membered rings) to the transmembrane region of the lipids seems to be a thermo-adaptive response, resulting in increased membrane packing and decreased membrane fluidity.

Archaeosomes (Figure 1) may be made using conventional methods (hydrated film sonicated, extrusion, and detergent dialysis) at any temperature in the physiological range or below, allowing thermally stable chemicals to be encapsulated. They can be produced and kept without degrading in the presence of air/oxygen. Archaeosomes are harmless and do not cause toxicity in mice, according to in vitro and in vivo investigations [3].

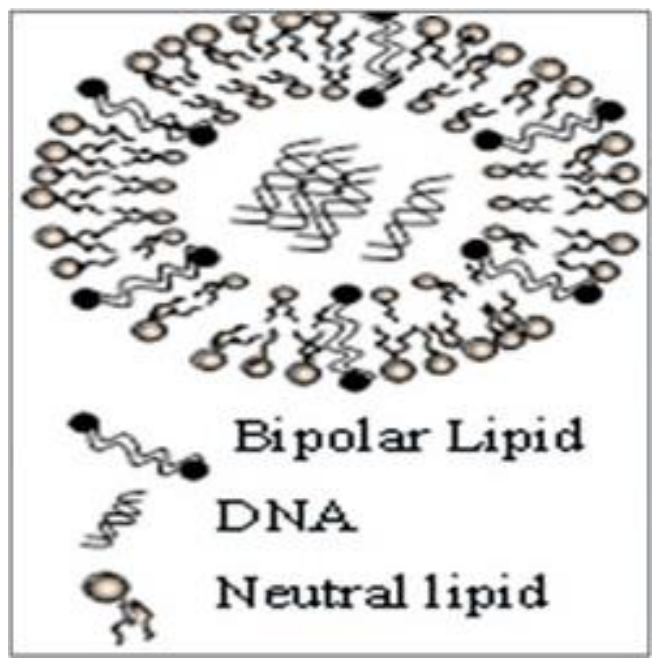

Figure 1. Structure of Archaeosomes. 


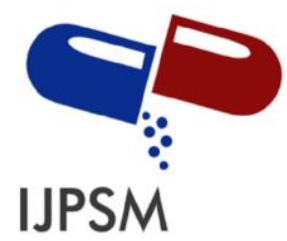

Kamini Kashyap, International Journal of Pharmaceutical Sciences and Medicine (IJPSM), Vol.6 Issue. 7, July- 2021, pg. 102-127

ISSN: 2519-9889

Impact Factor: 3.426

\section{SOURCE OF ARCHAEA}

Three major sources of archaea were found which will be very helpful for the availability for the same [4].

Halophiles: Halobacterium cutirubrum, Natronobacterium magadii.

Methanogens: Methanococcus voltae, Methanococcus jannaschii, Methanosaeta concilii, Methanosphaera stadtmanae, Methanobacterium espanolae, Methanospirillum hungatei, Methanosarcina mazei, Methanobrevibacter smithii.

Thermoacidophiles: Thermoplasma acidophilum

\section{ADVANTAGES OF ARCHAEAL LIPIDS}

Archaeal lipids play an important role from the production of pharmaceutical products to their stability maintainer. Some important advantages of archaeal lipids were described below [5]:

- The lipids found in archaea are more stable than those found in other phospholipids.

- The chemically modified archaeal lipid derivative improves the hydrolysis stability of other enzymes.

- Adding cyclic structure to lipids seems to be a thermo-adaptive response, resulting in increased membrane packing and decreased membrane fluidity.

- Saturated alkyl chains would enhance stability against oxidative degradation.

- It does not need the use of cholesterol in the formulation.

- The glycerol backbone's unique stereochemistry ensures resistance to phospholipases produced by other species.

- It may be used to target particular organs.

- Because bipolar lipids have improved stability, they may be produced and kept in the presence of air/oxygen without deterioration. 


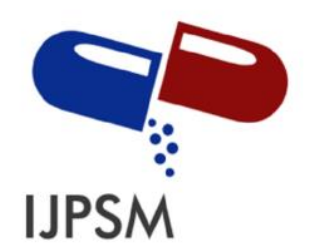

Kamini Kashyap, International Journal of Pharmaceutical Sciences and Medicine (IJPSM), Vol.6 Issue. 7, July- 2021, pg. 102-127

ISSN: 2519-9889

Impact Factor: 3.426

- Archaeosome formulations may be sterilized by autoclaving due to their excellent thermo-stability.

- In the environment, archaeal lipids are more thermo-labile.

- The absorption of phagocytic cells is higher, ensuring excellent adjuvant action.

- Archaeal lipids function as drug delivery systems that are self-adjuvanting.

\section{TYPES OF ARCHAEA LIPIDS}

Archaea lipids can be classified into three major categories as follows.

\section{Natural lipids}

Archaeal lipids have structures that are closely related to the species from which they are derived. Archaea may be divided into many species depending on their living conditions, and each has evolved its own set of lipids. Halophilic polar lipids (archaeal) are produced from monopolar dieter compounds, while acidophilic polar lipids are derived from bipolar tetraethers (caldarchaeol). However, most archaeal lipid membranes are made up of a mix of lipids with different kinds of polar heads (phosphate, glycerophosphate, sugar, and so on); furthermore, certain archaea species, such as methanogens, have evolved mixes of diether and tetraether type polar lipids. Natural archaeal lipids are isolated from typical Archaea species, covering the whole range of archaeal lipid structures. Total polar lipids (TPL) were isolated and purified from methanogens, halophiles, and thermoacidophiles by many research groups. Archaea were cultivated in ideal living circumstances before being extracted using organic solvents in large quantities. TPL were ultimately produced by precipitating a chloroform/methanol (2:1 v/v) solution with acetone. The resultant extracts were examined using thin-layer chromatography (TLC) and mass spectrometry (MS) and were either utilized directly or processed further using preparative TLC to identify pure polar lipid components. A glycerol moiety with two phytanyl chains on the sn-2,3 locations forms the basis of diether structures. Negatively charged phosphoethanolamine and phosphoglycerol are the primary polar head groups of such archaeal 


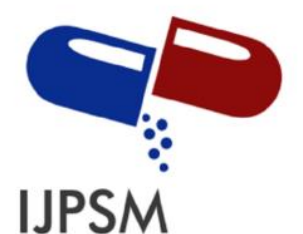

Kamini Kashyap, International Journal of Pharmaceutical Sciences and Medicine (IJPSM), Vol.6 Issue. 7, July- 2021, pg. 102-127

ISSN: 2519-9889 Impact Factor: $\mathbf{3 . 4 2 6}$

derivatives. The existence of two diphytanyl chains connected at both ends to two glycerol residues in an antiparallel (caldarchaeol) or parallel (isocaldarchaeol) way, while the glycerol configuration remains the same, characterizes tetraether components (Figure 2). Sugar neutral head groups, as well as negative phosphoinositol or zwitter ionic groups, may complete the polar lipid structure [6].

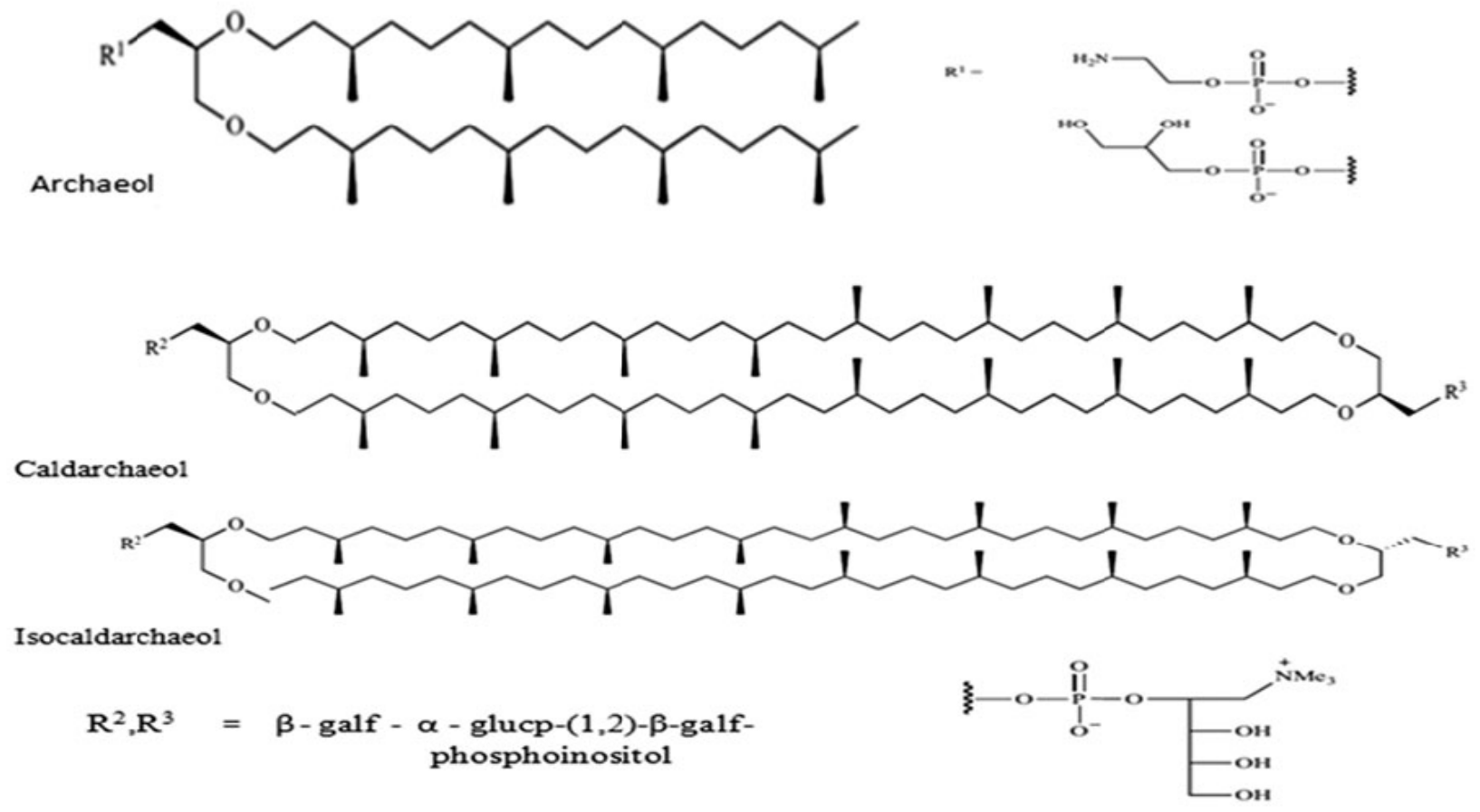

Figure 2. Structures of archaeal lipid.

\section{Chemically modified natural lipids}

Natural polar lipids from archaea lipid extracts typically have polar head groups that are susceptible to acidic hydrolysis (1 $\mathrm{M} \mathrm{HCl}-$ methanol). As a consequence of this chemical process, matching dihydroxyl archaeal lipid cores are isolated. Several novel structures of 


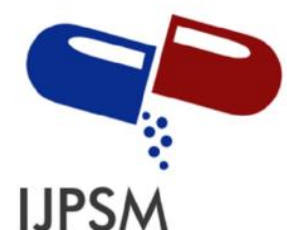

Kamini Kashyap, International Journal of Pharmaceutical Sciences and Medicine (IJPSM), Vol.6 Issue. 7, July- 2021, pg. 102-127

ISSN: 2519-9889 Impact Factor: $\mathbf{3 . 4 2 6}$

archaeal lipids obtained from T. acidophilum lipid extracts were patented by the Syrinx Diagnostika Company. Indeed, following hydrolysis of the original polar head groups, a few chemical synthesis processes such as oxidation of primary alcohols to carboxylic acids, activation, and coupling with appropriate amines provided easy access to the required archaeal lipids containing aminated polar head groups (Figure 3).

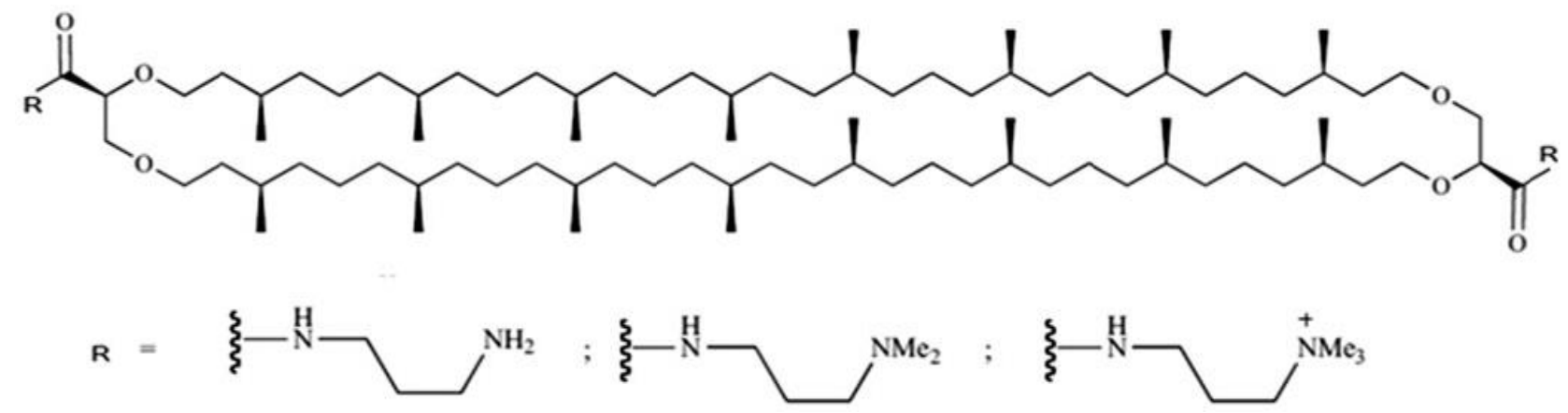

Figure 3. Aminated tetraethers.

Halobacterium salinarum and Tetrabacterium acidophilum provided the modified diether and tetraether lipid cores, respectively. Archaeols were then functionalized with sugar or phosphorylated head groups, while caldarchaeols were functionalized with the same polar head groups symmetrically or unsymmetrically. Sugars were selected from glucose di-, tri-, and tetrasaccharides, galactose, and mannose di-, tri-, and tetrasaccharides, phosphoserine, phosphoethanolamine, phosphoinositol, and phosphoglycerol were used to create phosphorylated groups [7] (Figure 4). 


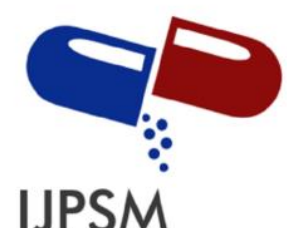

Kamini Kashyap, International Journal of Pharmaceutical Sciences and Medicine (IJPSM), Vol.6 Issue. 7, July- 2021, pg. 102-127

ISSN: 2519-9889

Impact Factor: $\mathbf{3 . 4 2 6}$
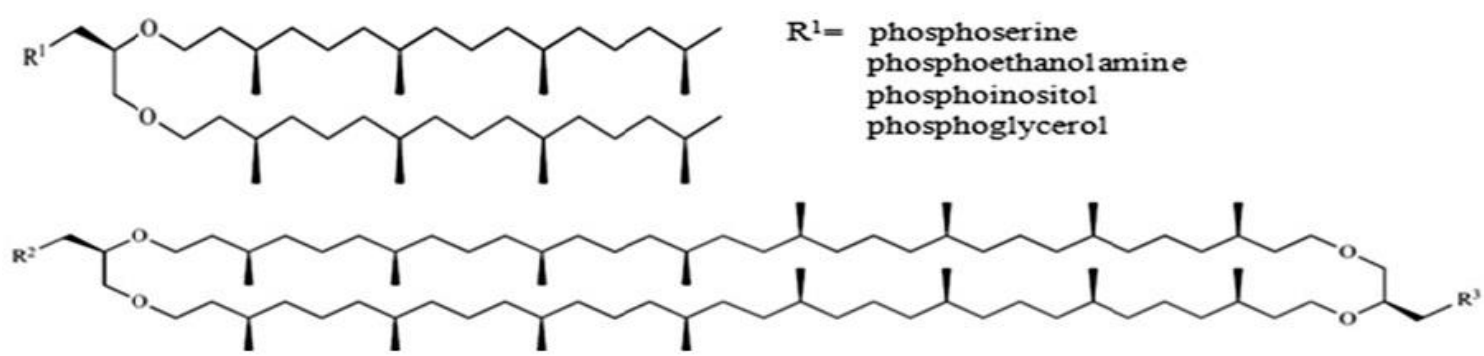

$\mathrm{R}^{2}, \mathrm{R}^{3}=\mathrm{GlC}-(1,6)-\mathrm{Glc}-$

Glc- $(1,4)-$ Glc-

Gal-(1,6)-Glc-

Gal- $(1,4)-G 1 c-$

Glc- $(1,4)-$ Glc- $(1,4)-G l c-$

Man-(1,2)-Man-(1,2)-Man-

Man-(1,2)-Man-(1,2)-Man-(1,2)-Man-

$\mathrm{R}^{2}, \mathrm{R}^{3}=$ phosphoserine

phosphoethanolamine

phosphoinositol

phosphoglycerol

Figure 4. Hemi synthetic diether and tetraether glycolipids.

\section{Totally synthetic lipids}

Although natural archaeal lipid extraction yielded large quantities of pure molecules with certain challenges, the complete synthesis of archaeal lipid analogs was explored by many academic or corporate research teams. A symmetrical 1,3-cyclopentane ring with two alkyl $\left(\mathrm{X}=\left(\mathrm{CH}_{2}\right)_{2}\right)$ or alkoxy $(\mathrm{X}=\mathrm{O})$ chains connected to two glycerol units form a variety of archaeal lipid analogs (Figure 5).
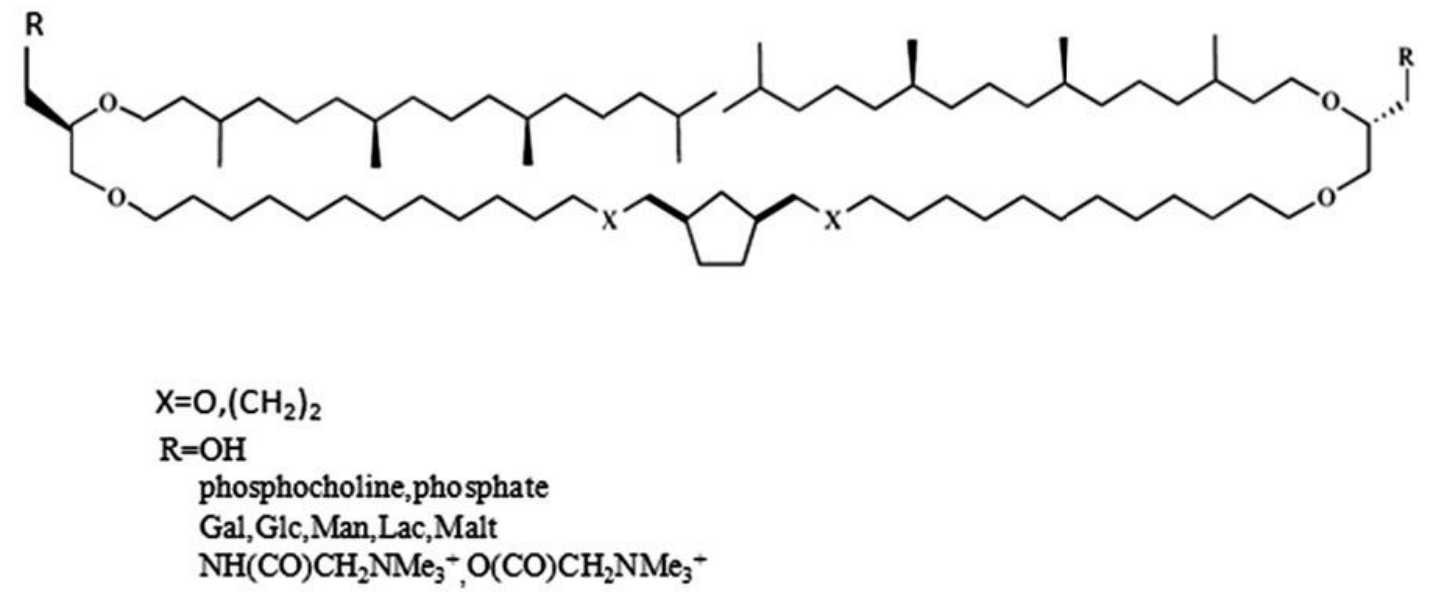

Figure 5. Synthetic archaeal lipid analog. 


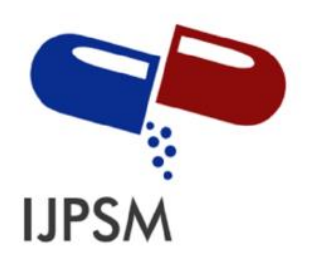

Kamini Kashyap, International Journal of Pharmaceutical Sciences and Medicine (IJPSM), Vol.6 Issue. 7, July- 2021, pg. 102-127

ISSN: 2519-9889

Impact Factor: 3.426

Finally, each glycerol residue has a phytanyl arm, resulting in a quasimacrocyclic tetraether structure. In comparison to natural archaeal lipids produced from isocaldarchaeol, the stereochemistry of the glycerol moieties was preserved (parallel arrangement of the glycerol groups). At both terminal ends of the tetraether core, a variety of polar head groups $(\mathrm{OH}$, sugars, glycine betaine, phosphorylated groups, PEG chains, and ligands) were inserted symmetrically or unsymmetrically. The $\mathrm{C}_{32}$ chain core of these tetraethers has two branching methyl groups in the center of the bridging chain. Two phytanyl or linear $\mathrm{C}_{16}$ arms resulted in quasimacrocyclic backbones in this case as well. The stereochemistry is identical to that of genuine archaeal lipids. Several polar head groups, such as a PEG chain, aminated groups, or phosphorylated groups, were used to construct and functionalize caldarchaeol and isocaldarchaeol analogs [8] (Figure 6).
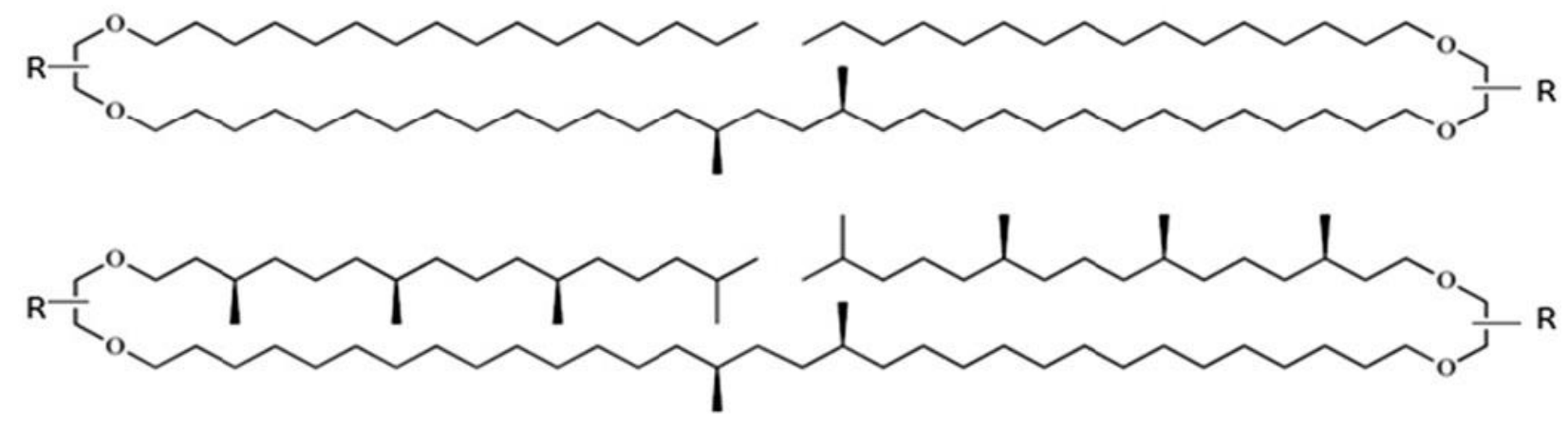

$\mathrm{R}=$ PEGylated chain, aminated groups, phosphate, phosphatidyl choline

Figure 6. Synthetic archaeal lipid analog.

\section{PREPARATION TECHNIQUES}

The extraction of lipids from appropriate archaeobacterial species has begun. TPL and neutral lipids like Squalene and other hydrocarbons make up the total natural archaeal lipid extract 


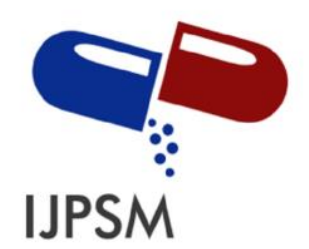

Kamini Kashyap, International Journal of Pharmaceutical Sciences and Medicine (IJPSM), Vol.6 Issue. 7, July- 2021, pg. 102-127

ISSN: 2519-9889

Impact Factor: 3.426

(TLE). TLE is obtained by extracting freeze-thawed biomass from chosen archaea species in chloroform/methanol/water. TPL and neutral lipids are isolated from TLE by precipitating TPL with acetone. These TPL synthesized from isoprenoid ether lipids with opposing sn-2,3 stereochemistry may be kept without additional conditions in chloroform or chloroform/methanol (2:1) solutions. The vesicle is made up entirely of glycol lipid sulfate and phospatidylglycerophosphate fraction. Chromatography, either column chromatography or preparative thin-layer chromatography, may be used to produce pure archaeal lipids. Chemical modifications may be made to the pure archaeal lipids to add particular head groups. The archaeal lipid phosphatidylmyoinositol is one polar group, while the other polar head group, either glucopyranose or galactopyranosyl glucopyranose, is required for structural stability. Furthermore, hydrating such lipids is very challenging. Using techniques established for the production of conventional liposomes, it was able to create archaeosome formulations and encapsulate/associate hydrophilic or hydrophobic molecules starting with natural, chemically modified, or synthetic archaeal lipids [9].

\section{Mechanical dispersion method}

This category includes all techniques that start with a lipid solution in an organic solvent and finish with lipid dispersion in water. The different components are usually mixed by codissolving the lipid in an organic solvent, then removing the organic solvent and hydrating the solid-lipid combination with an aqueous buffer. Archaeosomes are formed when lipids expand and hydrate spontaneously. At this stage, procedures change their final characteristics by including certain additional processing factors in different ways. Vortexing, sonication, freezethawing, and high-pressure extrusion are some of the post-hydration therapies [10]. 


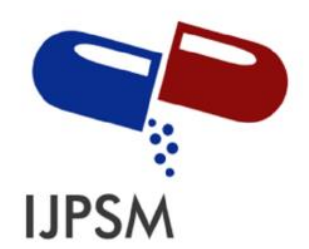

Kamini Kashyap, International Journal of Pharmaceutical Sciences and Medicine (IJPSM), Vol.6 Issue. 7, July- 2021, pg. 102-127

ISSN: 2519-9889

Impact Factor: 3.426

\section{Lipid hydration method}

Archaea lipid is dissolved in a solvent combination of chloroform-methanol (2:1) in a rotary evaporator flask, and a dried thin film of lipid is formed using the rotary evaporator. This procedure was carried out at room temperature $\left(30^{\circ} \mathrm{C}\right)$, at $60 \mathrm{rpm}$, and for $15 \mathrm{mins}$. Lipid hydration is accomplished in this method by adding $5 \mathrm{~mL}$ of saline phosphate buffer to the drug/antigen to be encapsulated. The consistent suspension was achieved using a rotary evaporator. At room temperature, archaeobacterial polar ether lipids are in a liquid crystallinelike or fluid form, allowing them to be hydrated, thus it was left at room temperature for 2 hrs to finish the swelling process. Multilamellar vesicles (MLVs) may be produced via this method. Even at freezing temperatures, archaeosomes will anneal. Thin-lipid film methods were used to make BMD-loaded archaeosomes and conventional liposomes, for example, utilizing archaeal lipids derived from Archaea $H$. salinarum and enhanced soy phosphatidylcholine, respectively. Vesicles composed of soybean phosphatidylcholine (SPC), sodium cholate (NaChol), and polar lipids from Halorubrum tebenquichense $(3: 1: 3 \mathrm{wt} / \mathrm{wt})$ are known as ultra deformable archaeosomes (UDA). For topical applications, UDA was produced using the lipid hydration technique [11].

\section{Membrane extrusion}

By gently passing prepared archaeosomes through a membrane filter with specified pore size, the size of the archaeosomes is decreased, and this may be done at a considerably lower pressure. In order to achieve high entrapment, the vesicles content is extruded with the dispersion media during the breaking and resealing of polar phospholipids as they travel through the polycarbonate membrane. Large unilamellar vesicles via extrusion (LUVETs) are the archaeosomes generated by this technique, and high lipid concentrations may achieve 30 percent encapsulation. Archaeosomes were made using the polar lipid methanol fraction. The dry lipid film was hydrated with $\mathrm{KPB}$ buffer $\left(250 \mathrm{nM}\right.$ sucrose, $10 \mu \mathrm{M}$ phosphate $\left(\mathrm{K}_{2} \mathrm{HPO}_{4} / \mathrm{KH}_{2} \mathrm{PO}_{4}\right)$, and $1 \mu \mathrm{M}$ 


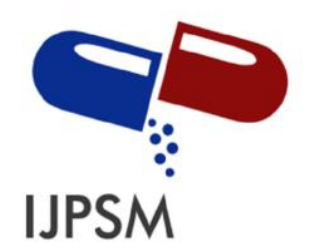

Kamini Kashyap, International Journal of Pharmaceutical Sciences and Medicine (IJPSM), Vol.6 Issue. 7, July- 2021, pg. 102-127

ISSN: 2519-9889

Impact Factor: $\mathbf{3 . 4 2 6}$

$\mathrm{MgCl}_{2}, \mathrm{pH} 7.4$, before being vortexed to produce multilamellar (ML) vesicles. Extrusion across a $400 \mathrm{~nm}$ membrane converted them into unilamellar (UL) vesicles [12].

\section{Freeze-thaw method}

In this technique, the UL dispersion is frozen for 15 mins before being thawed (melted) and subjected to a sonication cycle. The archaeosomes themselves merge and grow significantly in size. Then, by speeding up the pace at which packing errors are removed, sonication significantly decreases the permeability of the archaeosomes membrane. The sonication phase may be substituted with dialysis against hypo-osmolar buffer to produce gigantic vesicles with a diameter of $41 \mu \mathrm{m}$. The salt solution is combined with small unilamellar vesicles (SUVs), which are then frozen and thawed. As a consequence of the osmotic lysis, the huge vesicles produced by freeze-thawing expand and burst during dialysis, forming gigantic vesicles. The freezethawing technique was used to create archaeosomes made of polar lipid fraction E (PLFE) and traditional liposomes composed of EPC/cholesterol (3:2 molar ratio) for oral vaccination administration [13].

\section{Sonication}

By sonication at $60^{\circ} \mathrm{C}$, archaeosomes may be produced from the polar lipid fraction "PLF" of Sulfolobus solfataricus without the requirement for external lipid replenishment. Sulfolobus acidocaldarius polar lipids were successfully sonicated to generate archaeosomes at $0^{\circ} \mathrm{C}$. Sonication methods were used to make BMD-loaded archaeosomes and conventional liposomes, as well as archaeal lipids isolated from Archaea $H$. salinarum and enriched with phosphatidylcholine. For topical administration, sonicated vesicles were made by sonicating MLV dispersions at $80 \%$ amplitude for 4 min using a Hielscher UP50H ultrasonic disintegrator [14]. 


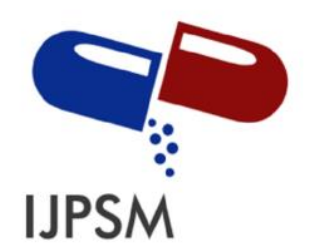

Kamini Kashyap, International Journal of Pharmaceutical Sciences and Medicine (IJPSM), Vol.6 Issue. 7, July- 2021, pg. 102-127

ISSN: 2519-9889

Impact Factor: $\mathbf{3 . 4 2 6}$

\section{French pressure cell extrusion}

After setting up the piston and pressure, filling the sample in the outlet hole, and turning on the power, a liquid sample of prefabricated MLVs is injected into the sample cavity. MLVs are extruded via a tiny aperture at ambient temperature $\left(40^{\circ} \mathrm{C}\right)$ and high pressure $(2000 \mathrm{psi})$, and then collected in a suitable container. This technique may be used to produce uni-lamellar or oligolamellar archaeosomes. For example, UL vesicles were made from ML vesicles by extrusion 10 times at the appropriate temperature $\left(65^{\circ} \mathrm{C}\right.$ for archaeosomes) through two-layered polycarbonate membranes (pore size $=200 \mathrm{~nm}$ ) under nitrogen gas pressure using a lipid extruder [15]

\section{Solvent dispersion method}

This technique involves dissolving lipids in an organic solution, which is then mixed with an aqueous phase containing the components to be entrapped inside the archaeosomes. The lipids arrange themselves at the organic-aqueous phase boundary, producing a lipid monolayer that makes up half of the archaeosome bilayer. The miscibility of the organic solvent and aqueous solution may be utilized to classify the methods employed in solvent dispersions. The organic solvent is miscible with the aqueous phase; the organic solvent is immiscible with the aqueous phase, and the latter is in excess; and the organic solvent is in excess and immiscible with the aqueous phase [16].

\section{Reverse phase evaporation}

Evaporation was used to remove the solvent from the emulsion in this technique. In general, polar lipids are dissolved in organic solvents and sonicated in a bath to produce an emulsion (w/o), which is then dried to a semisolid gel using a rotary evaporator under decreased pressure. Finally, LUVs may be created by strong mechanical shaking using a vortex mixer to produce a certain percentage of water droplets [17]. 


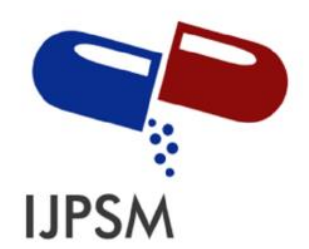

Kamini Kashyap, International Journal of Pharmaceutical Sciences and Medicine (IJPSM), Vol.6 Issue. 7, July- 2021, pg. 102-127

ISSN: 2519-9889

Impact Factor: 3.426

\section{Detergent dialysis method}

The ethereal phospholipids are brought into intimate contact with the aqueous phase in this technique using detergents as a mediator. Detergents form bonds with phospholipid molecules and help to screen the hydrophobic regions of the molecule from water [18]. Detergent reduction is achieved by four following approaches:

Dialysis: Dialysis may be done using either dialysis bags wrapped in big detergent-free buffers (equilibrium dialysis) or continuous flow cells.

Gel filtration: The detergent is rinsed away using size-exclusive chromatography in this technique. For gel filtration, any size filter (from Sephadex G-50 to Sephacryl S200-S1000) may be utilized. The archaeosomes are unable to pass through the pores of the beads packed in a column.

Adsorption using bio-beads: Shaking a mixed micelle solution with beaded organic polystyrene absorbers, such as XAD-2 beads and Bio-beads SM2, achieves detergent adsorption. The benefit of employing detergent absorbers is that they can remove detergents with a low critical micelle concentration that aren't fully removed by dialysis or gel filtration.

Dilution: The micellar size and polydispersity increase significantly when an aqueous mixed micellar solution of detergent and phospholipids is diluted with buffer, and as the system is diluted away from the mixed micellar phase boundary, an amorphous change from polydisperse micelles to monodisperse vesicles occurs. Due to the leaking of loaded molecules during the dialysis phase, the detergent/dialysis technique may result in poor trapping.

\section{HALLMARKS OF AN IDEAL ADJUVANT}

- Stability, bioavailability, and cost-effectiveness are all important considerations.

- Antibodies to autoimmune reactions are prevented.

- Non-pathological inflammatory responses are induced.

- Encouraging innate and acquired immune systems to communicate with one another. 


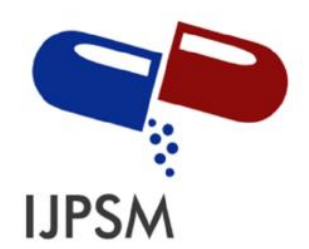

Kamini Kashyap, International Journal of Pharmaceutical Sciences and Medicine (IJPSM), Vol.6 Issue. 7, July- 2021, pg. 102-127

ISSN: 2519-9889

Impact Factor: 3.426

- Increased antibody response to a particular (humoral) antigen.

- Induction of a cell-mediated cytotoxic T-cell response.

- Activation of T-helper cells [19].

\section{APPLICATIONS OF ARCHAEOSOME FORMULATIONS}

\section{Self-adjuvanting drug delivery systems for cancer vaccines}

Strong CD4+ and CD8+ CTL responses to entrapped soluble antigens are induced by archaeosome adjuvants produced as archaeal ether glycolipid vesicles. Long-term tumor prevention requires CD8+ CTL responses in the host. In the absence of CD8+ T-cell cytotoxicity, spontaneous tumors grow in mice. Human CD8+ CTL responses to tumorassociated antigens have been proven to be very beneficial to patients, especially those with advanced cancer. As a result, there is a lot of interest in developing cancer immune treatments. The capacity of cancer vaccines to elicit a strong and suitable immune response is dependent on two important aspects: the identification of particular antigenic targets and the ability to elicit a robust and appropriate immunological response. The exogenous antigen is effectively delivered by archaeal ether glycerolipid vesicles (archaeosomes) for the development of humoral and cellmediated immunity. The potential of different archaeosomes lipid compositions to elicit a robust CD8+ CTL response to entrapped antigen was evaluated since the start of CD8+ cytotoxic T cells is essential for protective immunization against malignancies. A main (day 10) splenic CTL response was elicited when mice were immunized with ovalbumin (OVA) entrapped in all archaeosomes lipid compositions, suggesting processing for MHC class I presentation. The use of polar lipid compositions from Halophilic archaea to adjuvant this early CTL response was highly effective. By weeks 6-7, the lytic units had substantially decreased. Only M. smithii and T. acidophilum, which are both high in bipolar membrane-spanning caldarchaeols, were shown to elicit memory CTLs at 50 weeks. Mice immunized with OVA entrapped in $M$. smithii, $H$. salinarum, and T. acidophilum vesicles are protected against OVA-expressing solid tumor 


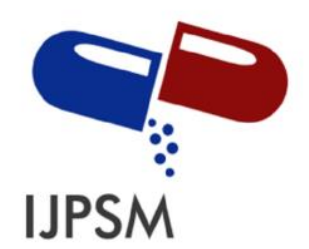

Kamini Kashyap, International Journal of Pharmaceutical Sciences and Medicine (IJPSM), Vol.6 Issue. 7, July- 2021, pg. 102-127

ISSN: 2519-9889

Impact Factor: $\mathbf{3 . 4 2 6}$

challenge for 6 weeks. Even a 3mg OVA dosage in archaeosomes slowed tumor development substantially. A therapeutic approach in which OVA-archaeosomes were administered concurrently with the tumor challenge resulted in tumor prevention. Innate therapeutic protection was given by antigen-free $T$. acidophilum archaeosomes that captured antigen $H$. salinarum archaeosomes. Vaccination with a CTL peptide epitope from the melanoma separation antigen, tyrosinase-related protein-2, in archaeosomes resulted in a protective CD8+ response against B16OVA metastases, suggesting that self-tumor antigens might be targeted. Archaea's lipid structural characteristics may affect primary, long-term, and/or innate immunity in various ways, influencing vaccination adjuvant selection [20].

\section{Immunoadjuvant for a vaccine against Chagas disease}

The protozoan parasite Trypanosoma cruzi causes American trypanosomiasis (Chagas sickness), a neglected tropical illness with a broad prevalence in Latin America. Globally, the WHO estimates that about 15 million people are infected. Clinical consequences of $T$. cruzi-induced heart disease afflict 50,000 children and adults each year, with many of them dying owing to a lack of appropriate therapy. Because of large-scale migrations, the illness has been found in nonendemic regions of the Americas and Europe, posing a significant risk of transmission. The development of innovative methods for the prevention and control of Chagas disease is critical. Vaccines and immunological treatments for $T$. cruzi infection are virtually non-existent at the moment. Several adjuvants have been tested to induce protective immunity against $T$. cruzi in conjunction with attempts to identify vaccine candidates, but with little success. The significant adjuvant effects of Archaeosomes have been shown by a growing body of research (ARC). Traditional liposomes are more actively absorbed by macrophages and antigen-presenting cells in vitro and in vivo than these vesicles surrounded by one or more bilayers produced with TPL derived from microorganisms belonging to the domain Archaea. They also vary from liposomes in that immune modulators are not required to enhance adjuvancy beyond a basic depot effect, 


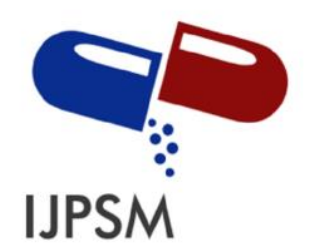

Kamini Kashyap, International Journal of Pharmaceutical Sciences and Medicine (IJPSM), Vol.6 Issue. 7, July- 2021, pg. 102-127

ISSN: 2519-9889

Impact Factor: $\mathbf{3 . 4 2 6}$

allowing for increased manufacturing scale. T. cruzi antigens may be effectively integrated into $\mathrm{ARC}$, and the resultant immunogen can prime a protective response against an intracellular parasite infection in mice after sc injection. In the development of future vaccines against this human disease, ARCs show promise as a safe and effective carrier adjuvant [21].

\section{As novel gene delivery systems}

In vitro gene transfection properties of novel cationic liposomes based on a mixture of neutral/cationic bilayer-forming lipids and archaeobacterial synthetic tetraether-type bipolar lipids have been demonstrated, and they represent a new approach for controlling the lipidic membrane fluidity of the complexes they form with DNA. In vitro gene transfection may be achieved using archaeobacterial lipids as cationic or co-lipids. The ability to modify the membrane characteristics of CL-DNA complexes by mixing traditional bilayer-forming lipids with monolayer-forming lipids has been discovered. Novel archaeoplexes' potential for in vivo gene transfection into the airway epithelium through nasal instillation or aerosolization, with the goal of lung-directed gene therapy for cystic fibrosis. Protein and peptide carriers for oral administration Because of the hostile GI environment, oral delivery of peptide and protein medicines is difficult. Peptides and proteins are better protected by lipid-based delivery methods. Archaeosomes are a unique lipid-based oral medication delivery system produced from PLFE isolated from $S$. acidocaldarius. In simulated GI fluids, archaeosomes had higher stability, allowing fluorescently tagged peptides to stay in the GI tract for longer after oral delivery. Despite the fact that archaeosomes had little impact on insulin transport across Caco-2 cell monolayers, in vivo studies revealed that archaeosomes containing insulin caused lower blood glucose levels than a standard liposome formulation. Under in vitro, archaeosomes made from PLFE were reasonably durable in simulated GI tract conditions, and in vivo, they aided the sluggish transit of fluorescently tagged peptides along the GI tract. In diabetic mice, archaeosomes outperformed standard liposomes as a carrier of oral insulin in lowering blood 


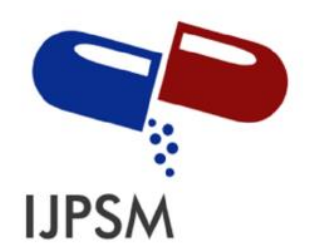

Kamini Kashyap, International Journal of Pharmaceutical Sciences and Medicine (IJPSM), Vol.6 Issue. 7, July- 2021, pg. 102-127

ISSN: 2519-9889

Impact Factor: $\mathbf{3 . 4 2 6}$

glucose levels. The hypoglycemic impact was moderate, which may be due to the formulation's low permeability of the intestinal epithelium after oral administration [22].

\section{As novel antigen delivery systems}

When the antigens were linked with liposomes comprised of either archaeal ether lipids or conventional lipids, the humoral immune response generated in BALB/c mice against bovine serum albumin or cholera toxin B subunit was compared. By encapsulating bovine serum albumin in archaeal lipid vesicles (archaeosomes) of approximately $200 \mathrm{~nm}$ diameter, antibody titers in sera from mice vaccinated intraperitoneally were raised to levels similar to those obtained with Freund's adjuvant. Six archaeosomes and three standard liposome formulations were found to be similar, indicating that archaeosomes were usually superior in potentiating an immune response. Furthermore, archaeosomes made consisting of polar lipids from M. smithii, a human colon bacterium, required just two vaccinations at most to reach close to the maximal antibody titer. $M$. smithii archaeosomes were revealed after a favorable reaction to presenting the more immunogenic cholera B subunit protein to the immune system of mice. To get the complete humoral reaction, the antigen has to be encapsulated in archaeosomes [23].

\section{Drug delivery systems for natural antioxidant compounds}

Multilamellar (MLVs) and unilamellar (SUVs) liposomes built up of archaeal polar lipids as a topical delivery method for natural antioxidant chemicals recovered from olive mill waste. SUVs were smaller than MLVs, with size values less than $200 \mathrm{~nm}$, which remained consistent throughout the stability testing. Traditional liposomes had a spherical shape, while archaeosomes had more uneven membranes, as shown by transmission electron microscopy. Both formulations had comparable vesicle encapsulation efficiency, which was sufficient to guarantee high antioxidant activity. One month after the formulations were prepared, stability tests were conducted, which revealed good stability with no change in the suspensions' early properties. 


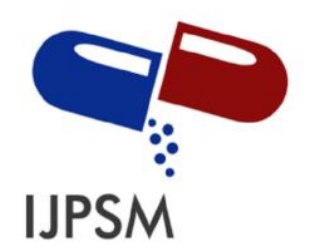

Kamini Kashyap, International Journal of Pharmaceutical Sciences and Medicine (IJPSM), Vol.6 Issue. 7, July- 2021, pg. 102-127

ISSN: 2519-9889

Impact Factor: $\mathbf{3 . 4 2 6}$

The potential of integrating liposomal suspensions in various excipients (Carbopol-940 and Pluronic-127) for topical administration was also investigated. In vitro diffusion experiments were carried out utilizing vertical diffusion Franz cells to evaluate the release behavior of the various systems produced. The vesicles were included into the gels and released after $24 \mathrm{hrs}$. Regardless of the excipient employed, archaeosome gels produced the same quantity of phenolic chemicals. Although there were significant variations in release between carbopol and pluronic gels in liposomal gels, Due to their stability, entrapment efficiency, and antioxidant activity, archaeosomes seemed to be a viable carrier for topical administration of antioxidant phenolic chemicals, which were similar to those produced with traditional phosphatidylcholine liposomes. Furthermore, archaeosomes seemed to be more flexible in terms of gel inclusion than ordinary liposomes. Thus, when utilizing archaeosomes, the release using carbopol or pluronic is quite comparable, allowing both excipients to be used interchangeably, while it does not occur when using traditional liposomes, allowing the choice of one excipient to be dependent on the desired effect [24].

\section{New drug carrier for delivery of Paclitaxel to breast cancer}

Paclitaxel-loaded archaeosomes decrease adverse effects and boost the therapeutic index of the drug. Carriers have made significant progress in the treatment of a variety of illnesses. Individual differences in lipid carriers are significant among carriers. One of the most significant lipid carriers is archaeosomes. Despite its therapeutic benefits, paclitaxel, a medication used to treat breast cancer, has certain undesirable side effects. Methanogenic archi bacteria were used to obtain archaeosomes, which were then produced in PBS with a specific ratio of Paclitaxel. The drug release of archaeosomes Paclitaxel was studied over a period of $26 \mathrm{hrs}$, and it was discovered that the most drug was released within the first three hrs. The cytotoxicity of archaeosomes Paclitaxel on a breast cancer cell line was assessed using the MTT test, which revealed that the cytotoxicity of archaeosomes Paclitaxel on a breast cancer cell line is higher 


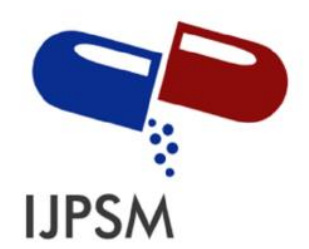

Kamini Kashyap, International Journal of Pharmaceutical Sciences and Medicine (IJPSM), Vol.6 Issue. 7, July- 2021, pg. 102-127

ISSN: 2519-9889

Impact Factor: $\mathbf{3 . 4 2 6}$

than the conventional Paclitaxel formulation. Paclitaxel's therapeutic index has been increased thanks to a new drug delivery method that uses archaeosomes [25].

\section{A carrier for topical delivery of BMD}

Archaeosomes, lipid vesicles produced from archaea polar lipids, and traditional phospholipid liposomes were used to assess the characteristics and potential of archaeosomes as new colloidal carriers for effective medication delivery to the skin. Thin-lipid film and sonication methods were used to create BMD-loaded archaeosomes and conventional liposomes, respectively, utilizing archaeal lipids derived from Archaea $H$. salinarum and enhanced with phosphatidylcholine. The size, zeta potential, entrapment effectiveness, and shape of vesicular formulations were all measured. In vitro drug permeation experiments through full-thickness pig skin were carried out employing Franz diffusion vertical cells, evaluating both archaeal and liposomal dispersions, to examine the impact of inclusion in two distinct colloidal carrier systems on the (trans) cutaneous delivery of medication. Archaeosomes proved to be the most efficient drug transporters, with significant drug penetration and accumulation in the skin stratum and epidermis. Archaeosomes have a lot of potential as a delivery mechanism for current applications. Incorporating archaeosomes into an anti-inflammatory drug's effectiveness in the treatment of skin diseases that need a local impact may be a new and promising approach [26].

\section{Archaeosomes delivery to different types of cargo into epithelial cells grown in vitro}

Archaeosomes are polar lipid-based liposomes made from archaeobacterial polar lipids. These contain unique structural characteristics that enhance lipid bilayer stability even in the presence of high temperatures, low or high $\mathrm{pH}$, phospholipases, and bile salts. As a result, they're ideal for the development of novel medication, gene, and vaccine delivery systems. Large UL archaeosomes (400 nm size) were produced from Aeropyrum pernix K1 and showed their promise as a foundation for the creation of an efficient and universal method for medication or 


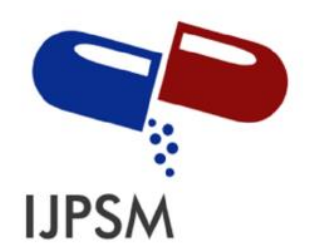

Kamini Kashyap, International Journal of Pharmaceutical Sciences and Medicine (IJPSM), Vol.6 Issue. 7, July- 2021, pg. 102-127

ISSN: 2519-9889

Impact Factor: $\mathbf{3 . 4 2 6}$

treatment administration to epithelial cells. Small fluorescent compounds (calcein), smaller proteins (60 kD like alisteriolysin), big protein aggregates (e.g. keratin 14), and plasmid DNA may all be delivered into epithelial cells grown in culture using these archaeosomes. Small molecules have a relatively high delivery efficacy, about $40 \%$, at this early stage of development. Even at large doses $(500 \mu \mathrm{g} / \mathrm{mL})$, prepared UL archaeosomes are not harmful to keratinocytes [27].

\section{UDA as topical adjuvant}

The skin has a large number of powerful antigen-presenting cells (APC) that are not easily accessible to parenteral vaccination unless via a challenging intradermal route. The topical approach is an appealing option since it allows for considerably closer contact with the APC on the skin. Topical vaccination offers more benefits than injectable vaccination, such as improved patient compliance, a lower risk of reinfection from contaminated material, and a lower need for specially trained people, sterilized equipment, and cold chain management. Dose variability and the requirement for powerful immunomodulators, such as bacterial ADP-ribosylating exotoxins, offset these benefits (cholera toxin, Escherichia coli, and their mutants). UDA are vesicles made from SPC, $\mathrm{NaCl}$, and $H$. tebenquichense polar lipids (3:1:3 w/w). UDL (composed of SPC and $\mathrm{NaChol}$ at a ratio of 6:1 w/w) and UDA were neither caught nor cytotoxic to keratinocytes; UDA was mainly captured by macrophages, with the potential of being decreased by $0.4-1.6 \mathrm{mg} / \mathrm{mL}$ phospholipids by 25-60\%. UDL was difficult to collect and did not produce any toxicity. Balb/C mice vaccinated topically with four doses of Ovalbumin (OVA)-loaded UDA at $75 \mu \mathrm{g}$ OVA/600 $\mu \mathrm{g}$ phospholipids (125 nm mean size and $42 \mathrm{mV}$ zeta potential) produce ten-fold to 100-fold greater IgG titers than those immunized with OVA-loaded UDL at the same dosage. Because UDA has a greater topical adjutancy and better phagocytic absorption due to its glycolipid content, both matrices penetrate to a comparable skin depth (almost $10 \mu \mathrm{m}$ after $1 \mathrm{hr}$ on human skin). Liposomes in the conventional sense merge and do not penetrate the intact skin of a mouse 


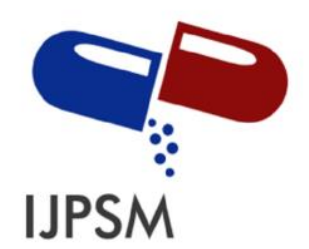

Kamini Kashyap, International Journal of Pharmaceutical Sciences and Medicine (IJPSM), Vol.6 Issue. 7, July- 2021, pg. 102-127

ISSN: 2519-9889

Impact Factor: 3.426

beyond a depth of $1 \mathrm{~mm}$. The lipid matrix of UDL, on the other hand, penetrates at least the depth of SC, bringing the aqueous content to the viable epidermis. Because of its unique mechanical behavior or ultra-deformability, UDL outperforms typical liposomes as topical adjuvants, thanks to the inclusion of edge activators such as $\mathrm{NaChol,} \mathrm{polysorbate,} \mathrm{or} \mathrm{ethanol} \mathrm{in}$ the phospholipid matrix. The UDL were lipids taken up by live skin cells after SC penetration. Archaeosomes provide effective adjuvants for the development of Th1, Th2, and CD8+ T cell responses to the entrapped soluble Ag after sc injection in mice [28].

\section{CONCLUSION}

Finally, we may conclude that the nano-carrier system described above has a great deal of potential for developing new, low-dose, and effective treatment methods for a variety of illnesses. The total number of clinical studies using archaeosomes and lipid-based products is an inspirational symbol. Several businesses are investing heavily in the creation and testing of archaeosome products for use in the treatment of a variety of illnesses. Archaeosomes have long been recognized as very effective carrier systems for delivering medicines, genes, and cells to their intended destinations. They are promising and may serve as a focus for future studies.

\section{CONFLICTS OF INTEREST}

No conflict of interest is declared.

\section{ACKNOWLEDGEMENT}

The author acknowledges the college management, principal, teachers, non-teaching staffs, and colleagues for their kind support.

\section{FUNDING INFORMATION}

No agency provided funds. 


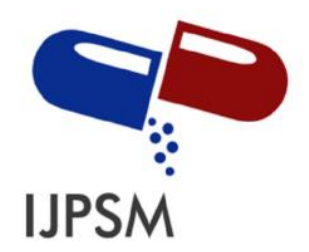

Kamini Kashyap, International Journal of Pharmaceutical Sciences and Medicine (IJPSM), Vol.6 Issue. 7, July- 2021, pg. 102-127

ISSN: 2519-9889

Impact Factor: 3.426

\section{REFERENCES}

[1]. Sprott GD, Tolson DL, Patel GB. Archaeosomes as novel antigen delivery systems. FEMS Microbiol Lett. 1997;154(1):17-22.

[2]. Li Z, Chen J, Sun W, Xu Y. Investigation of archaeosomes as carriers for oral delivery of peptides. Biochem Biophys Res Commun. 2010 2;394(2):412-7.

[3]. Patel GB, Sprott GD. Archaeobacterial ether lipid liposomes (archaeosomes) as novel vaccine and drug delivery systems. Crit Rev Biotechnol. 1999;19(4):317-57.

[4]. Kaur G, Garg T, Rath G, Goyal AK. Archaeosomes: an excellent carrier for drug and cell delivery. Drug Deliv. 2016;23(7):2497-512.

[5]. Higa LH, Schilrreff P, Perez AP, Iriarte MA, Roncaglia DI, Morilla MJ, Romero EL. Ultradeformable archaeosomes as new topical adjuvants. Nanomed Nanotechnol Biol Med. 2012;8(8):1319-28.

[6]. Li Z, Zhang L, Sun W, Ding Q, Hou Y, Xu Y. Archaeosomes with encapsulated antigens for oral vaccine delivery. Vaccine. 2011;29(32):5260-6.

[7]. Benvegnu T, Lemiègre L, Cammas-Marion S. New generation of liposomes called archaeosomes based on natural or synthetic archaeal lipids as innovative formulations for drug delivery. Rec Pat Drug Deliv Formul. 2009;3(3):206-20.

[8]. Réthoré G, Montier T, Le Gall T, Delepine P, Cammas-Marion S, Lemiègre L, Lehn P, Benvegnu T. Archaeosomes based on synthetic tetraether-like lipids as novel versatile gene delivery systems. Chem Commun. 2007(20):2054-6.

[9]. Gonzalez RO, Higa LH, Cutrullis RA, Bilen M, Morelli I, Roncaglia DI, Corral RS, Morilla MJ, Petray PB, Romero EL. Archaeosomes made of Halorubrum tebenquichense total polar lipids: a new source of adjuvancy. BMC Biotechnol. 2009;9(1):1-2. 


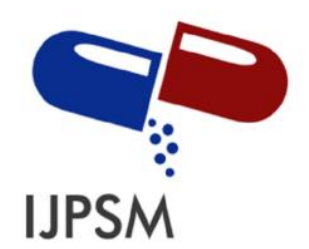

Kamini Kashyap, International Journal of Pharmaceutical Sciences and Medicine (IJPSM), Vol.6 Issue. 7, July- 2021, pg. 102-127

ISSN: 2519-9889

Impact Factor: 3.426

[10].Krishnan L, Sad S, Patel GB, Sprott GD. The potent adjuvant activity of archaeosomes correlates to the recruitment and activation of macrophages and dendritic cells in vivo. J Immunol. 2001;166(3):1885-93.

[11].Krishnan L, Dennis Sprott G. Archaeosomes as self-adjuvanting delivery systems for cancer vaccines. J Drug Targ. 2003;11(8-10):515-24.

[12].González-Paredes A, Clarés-Naveros B, Ruiz-Martínez MA, Durbán-Fornieles JJ, Ramos-Cormenzana A, Monteoliva-Sánchez M. Delivery systems for natural antioxidant compounds: Archaeosomes and archaeosomal hydrogels characterization and release study. Int J Pharmaceut. 2011;421(2):321-31.

[13].Patel GB, Zhou H, KuoLee R, Chen W. Archaeosomes as adjuvants for combination vaccines. J Liposome Res. 2004;14(3-4):191-202.

[14].Sprott GD, Sad S, Fleming LP, DiCaire CJ, Patel GB, Krishnan L. Archaeosomes varying in lipid composition differ in receptor-mediated endocytosis and differentially adjuvant immune responses to entrapped antigen. Archaea. 2003;1(3):151-64.

[15].Krishnan L, Sad S, Patel GB, Sprott GD. Archaeosomes induce enhanced cytotoxic T lymphocyte responses to entrapped soluble protein in the absence of interleukin 12 and protect against tumor challenge. Cancer Res. 2003;63(10):2526-34.

[16].Attar A, Ogan A, Yucel S, Turan K. The potential of archaeosomes as carriers of pDNA into mammalian cells. Artif cells Nanomed Biotechnol. 2016;44(2):710-6.

[17].Higa LH, Corral RS, Morilla MJ, Romero EL, Petray PB. Archaeosomes display immunoadjuvant potential for a vaccine against Chagas disease. Human Vaccin Immunother. 2013;9(2):409-12.

[18].González-Paredes A, Manconi M, Caddeo C, Ramos-Cormenzana A, MonteolivaSánchez M, Fadda AM. Archaeosomes as carriers for topical delivery of betamethasone dipropionate: in vitro skin permeation study. J Liposome Res. 2010;20(4):269-76. 


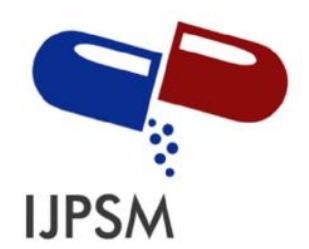

Kamini Kashyap, International Journal of Pharmaceutical Sciences and Medicine (IJPSM), Vol.6 Issue. 7, July- 2021, pg. 102-127

ISSN: 2519-9889

Impact Factor: $\mathbf{3 . 4 2 6}$

[19].Zavec AB, Ota A, Zupancic T, Komel R, Ulrih NP, Liovic M. Archaeosomes can efficiently deliver different types of cargo into epithelial cells grown in vitro. J Biotechnol. 2014;192:130-5.

[20].Moghimipour E, Kargar M, Handali S. Archaeosomes as means of nano-drug delivery. Rev Med Microbiol. 2014;25(2):40-5.

[21]. Attar A, Bakir C, Yuce-Dursun B, Demir S, Cakmakci E, Danis O, Birbir M, Ogan A. Preparation, characterization, and in vitro evaluation of isoniazid and rifampicin-loaded archaeosomes. Chem Biol Drug Des. 2018;91(1):153-61.

[22].Rezelj S, Kozorog M, Švigelj T, Ulrih NP, Žnidaršič N, Podobnik M, Anderluh G. Cholesterol enriched archaeosomes as a molecular system for studying interactions of cholesterol-dependent cytolysins with membranes. J Membr Biol. 2018;251(3):491-505.

[23].Omri A, Makabi-Panzu B, Agnew BJ, Sprott GD, Patel GB. Influence of coenzyme Q10 on tissue distribution of archaeosomes, and pegylated archaeosomes, administered to mice by oral and intravenous routes. J Drug Targ. 1999;7(5):383-92.

[24].Higa LH, Arnal L, Vermeulen M, Perez AP, Schilrreff P, Mundiña-Weilenmann C, Yantorno O, Vela ME, Morilla MJ, Romero EL. Ultradeformable archaeosomes for needle free nanovaccination with Leishmania braziliensis antigens. PLoS One. 2016;11(3):e0150185.

[25].Napotnik TB, Valant J, Gmajner D, Passamonti S, Miklavčič D, Ulrih NP. Cytotoxicity and uptake of archaeosomes prepared from Aeropyrum pernix lipids. Human Exp Toxicol. 2013;32(9):950-9.

[26].Šuštar V, Zelko J, Lopalco P, Lobasso S, Ota A, Ulrih NP, Corcelli A, Kralj-Iglič V. Morphology, biophysical properties and protein-mediated fusion of archaeosomes. PLoS One. 2012 Jul 6;7(7):e39401. 


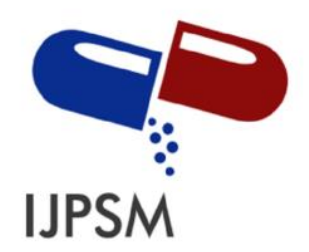

Kamini Kashyap, International Journal of Pharmaceutical Sciences and Medicine (IJPSM), Vol.6 Issue. 7, July- 2021, pg. 102-127

ISSN: 2519-9889

Impact Factor: $\mathbf{3 . 4 2 6}$

[27].Jiblaoui A, Barbeau J, Vivès T, Cormier P, Glippa V, Cosson B, Benvegnu T. Folateconjugated stealth archaeosomes for the targeted delivery of novel antitumoral peptides. RSC Adv. 2016;6(79):75234-41.

[28].Krishnan L, Dicaire CJ, Patel GB, Sprott GD. Archaeosome vaccine adjuvants induce strong humoral, cell-mediated, and memory responses: comparison to conventional liposomes and alum. Infect Immun. 2000;68(1):54-63.

\section{About Author:}

\section{Kamini Kashyap}

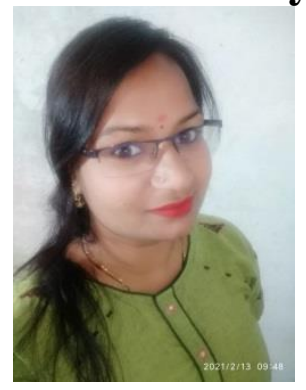

Department of Pharmaceutics, School of Pharmacy, Chouksey College of Engineering, NH49, Masturi - Jairamnagar Road, Lalkhadan, Bilaspur 495004, Chhattisgarh, India kashyapkamini127@gmail.com

Phone: +91-9109932032 